\title{
Is there a new role for angiotensin-converting-enzyme inhibitors in elderly patients?
}

\section{Ann Cranney MD MSc}

\author{
$\infty \quad$ See related article page 867
}

$\mathrm{T}$ he preservation of functional independence in elderly people depends on the ability to maintain muscle mass, strength and balance. Sarcopenia refers to the age-related declines in muscle mass and function and is associated with an increased risk of falls, fractures and frailty. ${ }^{1}$ The cause of sarcopenia is multifactorial, and suggested mechanisms include declines in local growth factors (e.g., insulin-like growth factor), mitochondrial dysfunction, muscle-fibre atrophy and the loss of functioning motor units. ${ }^{1,2}$ Various interventions have been used to target the decline in muscle strength in elderly people. Resistance exercise training and the use of nutritional supplementation have been shown to significantly improve muscle mass and function. ${ }^{3,4}$ However, it is often difficult for elderly people to sustain these types of exercise programs, which has prompted the exploration of other interventions.

As summarized by Sumukadas and colleagues in this issue, ${ }^{5}$ multiple sources of evidence support the role of angiotensin-converting-enzyme (ACE) inhibition in the maintenance of muscle strength and physical function in elderly people. ${ }^{5}$ For example, the use of ACE inhibitors in the treatment of congestive heart failure has been associated with reduced mortality ${ }^{6}$ and the prevention of declines in physical function. ${ }^{7}$ A previous study involving patients with heart failure demonstrated that the ACE inhibitor perindopril improved the participants' 6-minute walking distance. ${ }^{8}$ Whether this benefit is related to a direct effect of ACE inhibition on cardiac function, via improvements in endothelial function, or whether it is mediated by effects on nitric oxide or insulin-like growth factors on skeletal muscle is unknown. ${ }^{9}$ Observational studies involving elderly patients with hypertension who had no heart failure have reported slower declines in muscle strength and improved physical performance among patients taking ACE inhibitors compared with those taking other antihypertensive medications. ${ }^{7,10}$ People with the II genotype of the ACE gene have low ACE levels and have been found to have improved endurance and muscle strength. ${ }^{11}$ These findings suggest that ACE inhibitors improve muscle strength by mimicking the ACE II genotype effects. ${ }^{11,12}$

To evaluate the hypothesis that ACE inhibitors can result in improvements in muscle function and exercise capacity in elderly people with functional impairment, Sumukadas and colleagues completed a 20-week randomized controlled trial of perindopril versus placebo. Both the participants and the investigators were blinded to group allocation. The participants were 65 years or older (mean age 78.6 years) and had self-reported problems with mobility or activities of daily liv-

\section{Key points of the article}

- Age-related declines in muscle mass and quality (sarcopenia) are associated with significant morbidity and mortality

- In the randomized controlled trial by Sumukadas and colleagues, the angiotensin-converting-enzyme (ACE) inhibitor perindopril was found to improve the exercise capacity and prevent declines in health-related quality of life among functionally impaired elderly people

- There was a trend toward a reduction in falls; however, patients with symptomatic hypotension were excluded from the trial, and only $24 \%$ of patients screened met the inclusion criteria

- Research is required to confirm the results of this trial and to determine whether the observed improvements in exercise capacity persist over time

- Additional research is needed into the exact mechanisms of action of ACE inhibitors on physical function and healthrelated quality of life

ing. Twenty percent of the participants had a history of ischemic heart disease, but none had evidence of left ventricular dysfunction or congestive heart failure. Forty-eight percent used walking aids, and the median Mini-Mental State Examination score was 29 (of 30 ).

The primary outcome in the trial by Sumukadas and colleagues was the change in the 6-minute walking distance over the study period. The authors found that, compared with placebo, perindopril resulted in significant improvements in the 6-minute walking distance from baseline to 20 weeks, equivalent to the improvement seen after 6 months of exercise. Another important finding in the perindopril group was the maintenance of health-related quality of life, as assessed using the EuroQol EQ-5D questionnaire. However, the 2 groups did not differ significantly in the change in times recorded for the sit-to-stand test or the timed up-and-go test. These tests are used to predict falls or mobility problems in elderly people living in the community. The lack of improvement in these outcomes may reflect either the baseline level of impairment of the study cohort or the inability of these measures to detect change in this population. A self-reported history of falls was recorded at baseline, and falls that occurred during the study were noted as adverse events, owing

Ann Cranney is with the Ottawa Health Research Institute, Ottawa, Ont. 
Box 1: Potential mechanisms of action of angiotensinconverting-enzyme (ACE) inhibitors on physical function

- Improvement of cardiac and vascular function

- Increased nitric oxide production, which may improve skeletal muscle contractility and increase the number of sarcomeres (decreased degradation of bradykinin)

- Prevention of age-related mitochondrial dysfunction

- Shift from type II to type I muscle fibres

to the concern that ACE inhibitors may increase the risk of falls. The authors found a trend toward a reduction in the number of falls reported during the study period in the perindopril group relative to the placebo group, with 8 patients reporting falls in the treatment group and Io patients in the placebo group.

With respect to potential adverse events, the significant increases in serum potassium and creatinine levels and declines in blood pressure seen in the perindopril group relative to the placebo group highlight the need for monitoring if this drug is to be used in clinical practice and future clinical trials.

Another important issue highlighted by this trial is the difficulty in recruiting elderly study participants. ${ }^{13}$ Only $24 \%$ of those screened met the eligibility criteria for the trial, and only $21 \%$ of those who were eligible agreed to participate. In addition, patients with symptomatic hypotension were excluded, which has implications for the applicability of these results to clinical practice.

Sumakadas and colleagues should be congratulated on moving this area of research forward. Their results are promising and lend support to the hypothesis that ACE inhibition has a positive effect on physical function, possibly through effects on skeletal muscle. Their findings need to be confirmed in a larger trial, ideally one that measures functional outcomes, such as the timed up-and-go test, a patient-centred outcome measure of function and the risk of falls. The use of more stringent eligibility criteria with respect to baseline functional impairment, such as a history of falls within the past year or treatment for balance problems, would also be useful. Other outcomes could include changes in body composition, such as lower extremity muscle mass. Given the challenges in recruiting elderly participants, future trials may need to be either multicentred or pragmatic trials that evaluate relevant clinical outcomes using less rigorous methods for data collection.

For the clinician considering how to manage their elderly hypertensive patients who have functional or mobility impairment, the benefits noted in the trial by Sumukadas and colleagues provide further rationale for selecting an $\mathrm{ACE}$ in- hibitor, especially for patients who have difficulty participating in an exercise program.

Future research should be directed at improving our understanding of the mechanism of action of ACE inhibitors on physical function (Box I). It would be important to evaluate the relative effectiveness of ACE inhibitors compared with other interventions such as resistance exercise or nutritional supplementation. It would also be useful to confirm whether ACE inhibitors would benefit all elderly people with functional impairment or whether it would be more beneficial in specific subgroups (e.g., individuals under 80 years and those with peripheral vascular disease). If these findings are confirmed, they could have important public health implications, since ACE inhibitors could then be used to delay declines in muscle strength, physical function and health-related quality of life.

Competing interests: None declared.

Acknowledgement: Ann Cranney is the recipient of a Canadian Institutes of Health Research Investigator Award.

\section{REFERENCES}

I. Di Iorio A, Abate M, Di Renzo D, et al. Sarcopenia: age-related skeletal muscle changes from determinants to physical disability. Int J Immunopathol Pharmacol 2006;19:703-19.

2. Marzetti E, Leeuwenburgh C. Skeletal muscle apoptosis, sarcopenia and frailty at old age. Exp Gerontol 2006;4I:I234-8.

3. Melov S, Tarnopolsky MA, Beckman K, et al. Resistance exercise reverses aging in human skeletal muscle. PLOS ONE 2007;2:e465.

4. Candow DG, Chilibeck PD. Effect of creatine supplementation during resistance training on muscle accretion in the elderly. J Nutr Health Aging 2007;II:185-8.

5. Sumukadas D, Witham MD, Struthers AD, et al. Effect of perindopril on physical function in elderly people with functional impairment: a randomized controlled trial. CMAJ 2007; 177:867-74.

6. Yusuf S, Sleight P, Pogue J, et al. Effects of an angiotensin-converting-enzyme inhibitor, ramipril, on cardiovascular events in high-risk patients. The Heart Outcomes Prevention Evaluation Study Investigators [published errata in $N$ Engl J Med 2000;342:748 and 2000;342:1376]. N Engl J Med 2000;342:I45-53.

7. Onder G, Penninx BW, Balkrishnan R, et al. Relation between use of angiotensinconverting enzyme inhibitors and muscle strength and physical function in older women: an observational study. Lancet 2002;359:926-30.

8. Hutcheon SD, Gillespie ND, Crombie IK, et al. Perindopril improves six minute walking distance in older patients with left ventricular systolic dysfunction: a randomised double blind placebo controlled trial. Heart 2002;88:373-7.

9. Maggio M, Ceda GP, Lauretani F, et al. Relation of angiotensin-converting enzyme inhibitor treatment to insulin-like growth factor-I serum levels in subjects $>65$ years of age (the InCHIANTI study). Am J Cardiol 2006;97:1525-9.

Io. Carter CS, Onder G, Kritchevsky SB, et al. Angiotensin-converting enzyme inhibition intervention in elderly persons: effects on body composition and physical performance. J Gerontol A Biol Sci Med Sci 2005;60:1437-46.

II. Williams AG, Rayson MP, Jubb M, et al. The ACE gene and muscle performance. Nature 2000;403:614.

I2. Sumukadas D, Struthers AD, McMurdo ME. Sarcopenia - A potential target for angiotensin-converting enzyme inhibition? Gerontology 2006;52:237-42.

13. McMurdo ME, Witham MD, Gillespie ND. Including older people in clinical research. BMJ 2005;331:1036-7.

Correspondence to: Dr. Ann Cranney, Clinical Epidemiology Program, Ottawa Health Research Institute, Rm. ASB I-oog, I053 Carling Ave., Ottawa ON KIY 4E9; ancranney@ohri.ca 